\title{
Perturbation Realization, Potentials, and Sensitivity Analysis of Markov Processes
}

\author{
Xi-Ren Cao, Fellow, IEEE, and Han-Fu Chen, Fellow, IEEE
}

\begin{abstract}
Two fundamental concepts and quantities, realization factors and performance potentials, are introduced for Markov processes. The relations among these two quantities and the group inverse of the infinitesimal generator are studied. It is shown that the sensitivity of the steady-state performance with respect to the change of the infinitesimal generator can be easily calculated by using either of these three quantities and that these quantities can be estimated by analyzing a single sample path of a Markov process. Based on these results, algorithms for estimating performance sensitivities on a single sample path of a Markov process can be proposed. The potentials in this paper are defined through realization factors and are shown to be the same as those defined by Poisson equations. The results provide a uniform framework of perturbation realization for infinitesimal perturbation analysis (IPA) and non-IPA approaches to the sensitivity analysis of steady-state performance; they also provide a theoretical background for the $P A$ algorithms developed in recent years.
\end{abstract}

Index Terms-Perturbation analysis, Poisson equations, samplepath analysis.

\section{INTRODUCTION}

$\mathbf{T}$ HE SINGLE sample path-based performance sensitivity analysis of discrete-event system has become an increasingly important area. The main objective of this research area is to obtain performance sensitivities with respect to system parameters by analyzing a single sample path of a discreteevent system. The area is promising because of its practical usefulness. First, the single sample path-based techniques save a great amount of computation in simulation for system optimization; second and perhaps more importantly, they can be applied to on-line performance optimization of real world systems where changing the values of parameters is infeasible.

One of the major research fields in this area is perturbation analysis (PA), which takes a dynamic point of view in studying the system behavior. The history of the development of PA resembles those of many other scientific fields; after an initial period of exploration of the common nature of some practical problems, a mathematical formulation was developed [5]. With

Manuscript received January 2, 1996; revised May 15, 1997. Recommended by Associate Editor, E. K. P. Chong. X.-R. Cao was supported in part by Hong Kong UPGC under Grant HKUST 599/94E. H.-F. Chen was supported by the National Natural Science Foundation of China and the Hong Kong University of Science and Technology.

X.-R. Cao is with The Hong Kong University of Science and Technology, Clear Water Bay, Kowloon, Hong Kong. He is currently on leave at AT\&T Laboratories, Holmdel, NJ 07733 USA.

H.-F. Chen is with the Institute of Systems Science, The Chinese Academy of Sciences, Beijing 100080, China.

Publisher Item Identifier S 0018-9286(97)07624-1. this formulation, it became clear that the simple PA algorithms developed earlier (called infinitesimal perturbation analysis (IPA), hereafter) in fact yield "sample derivatives"; although these sample derivatives are unbiased or strongly consistent for many systems, it is not true for many others [6], [28]. This insight has set up two fundamental research directions: to establish IPA theory, including the proof of convergence of IPA algorithms for various discrete-event systems, and to develop new algorithms for systems where IPA does not work well. After the hard work of many researchers in more than one decade, fundamental results have been obtained for IPA [29], [22], [7], [12], [14], [47]-[49], and many useful techniques that apply to different cases where IPA fails have been proposed [1], [13], [17], [19], [21], [25], [30], [31], [50]. By and large, the theory for IPA is relatively mature, but the works beyond IPA still seem exploratory; a general approach that is simple and applies to a wide class of problems has yet to be developed.

Besides the fundamental works in developing theory and algorithms, PA has been successfully applied in a number of practical engineering problems [2], [11], [26], [51], [52].

In this paper, we study the sensitivity of the steady-state performance of a Markov process with respect to its infinitesimal generator. It is well known that IPA yields an estimate of zero for the performance sensitivity (see, e.g., [6], [28], and [29]), which is apparently useless. Our goal is to develop a simple approach that provides accurate estimates. The approach is widely applicable because a Markov process is the most fundamental model for many discrete-event systems. The work was motivated by some recent developments in the area [16], [17], [9]; it may be also related to [19] and [20]. We first introduce a concept, perturbation realization, and define a quantity, realization factor, for studying the effect of state changes in a Markov process on its performance. This can be considered as an extension of the perturbation realization theory [7] from infinitesimal perturbations to finite perturbations. We prove that under some minor conditions the realization factors can be determined uniquely by a Lyapunov equation. Next, using the realization factors, we introduce the concept of potentials and establish some simple relationships among potentials, realization factors, and the group inverse of the infinitesimal generator. We show that the performance sensitivity can be calculated by using any of these three equivalent quantities. Furthermore, we develop some formulas that allow us to estimate these quantities conveniently with a single sample path of the Markov process. Finally, we show 
that the potentials defined in this paper through realization factors are in fact the same as those defined by Poisson equations in the literature (see, e.g., [24], [27], [34], [35], [37]-[39], and [41]). The results for Markov chains are briefly stated as special cases.

We emphasize the main contributions of this paper with the following points.

1) We developed some algorithms for finite perturbations which are almost as simple as IPA yet yield very accurate estimates. As shown in (16) and (17), the performance derivatives can be calculated by using potentials or realization factors; from (5) and (25), these quantities can be estimated based on a single sample path. The calculation involved in the estimation is only addition for Markov chains and linear integration for Markov processes; thus, the computations required are comparable to IPA estimates. The detailed algorithms are worked out in another paper [10].

Since a Markov process is the basic model for many discrete-event systems, it is conceivable that similar algorithms can be developed for many other systems such as queueing networks and generalized semi-Markov processes. Thus, the results may be viewed as one important step toward a solution to the long standing problem: finding a simple and generally applicable technique that provides accurate estimates for performance sensitivities when IPA fails.

2) The concept of perturbation realization, which was successfully used in IPA to study the sensitivity of steadystate performance, is extended beyond IPA, where a small change in parameter may result in a big change in the system's sample paths. This provides a unified framework for both IPA and non-IPA approaches (see the discussion after Theorem 4 for an explanation). It also provides a theoretical background for the PA algorithms developed earlier in [16], [17], [19], and [20].

3) The results contribute to the modern Markov theory by relating the potentials (with discount rate $\alpha=0$ ) to perturbation realization and the single sample pathbased sensitivity analysis. Theorem 3 provides a nice interpretation of the solutions to Poisson equations, and our results relate the potentials to realization factors that can be interpreted as the fundamental elements of sensitivity analysis.

We discuss Markov processes with a countable state space; the performance functions are not required to be bounded. In Section II, the sensitivity analysis problem is described. In Section III, the realization factor is defined, the Lyapunov equation that uniquely determines realization factors is derived, and two theorems for estimating the realization factors are proved. In Section IV, the group inverse of a matrix is first reviewed, the concept of performance potentials are formally introduced by using realization factors, and the sensitivity formula is obtained by using group inverse, realization factors, or performance potentials. The relations among these quantities and the Poisson equation are discussed. In Section V, similar results are listed for Markov chains. The paper concludes with a short discussion in Section VI.

\section{THE PROBLEM}

Consider a positive recurrent and irreducible (ergodic) Markov process $X=\left\{X_{t}, t \geq 0\right\}$ with a countable state space $\mathcal{E}=\{1,2, \cdots\}$ and an infinitesimal generator $A=\left[a_{i j}\right]$, where $a_{i j} \geq 0, i \neq j, a_{i i}<0$. We assume that $a_{i j}, i, j \in \mathcal{E}$, are bounded, i.e., there is $K<\infty$ such that $\left|a_{i j}\right|<K$ for all $i, j \in \mathcal{E}$. Thus, the Markov process is regular. (The condition that $\left|a_{i j}\right|, i, j \in \mathcal{E}$, are bounded is stronger than regularity. For example, the $\mathrm{M} / \mathrm{M} / \infty$ queue is regular, but the corresponding $\left|a_{i j}\right|$ are not bounded.) Denote by $\pi=\left(\pi_{1}, \pi_{2}, \cdots\right)$ the row vector representing the steady-state probabilities of the Markov process. Let $e=(1,1, \cdots)^{\prime}$, where the prime represents the transpose. We have $\pi e=1$ and

$$
A e=0, \quad \pi A=0 \text {. }
$$

Let $M=\left[m_{i j}\right]$ with $m_{i j}$ being the mean first passage time from state $i$ to state $j$. A Markov process is said to be strong ergodic, if $\pi M$ is finite [34]. In Section IV, we require the Markov process be strong ergodic.

We first normalize the Markov process (see, e.g., [18]). Let $\bar{A}=(1 / K) A$, i.e., $\bar{a}_{i j}=a_{i j} / K$, for all $i, j \in \mathcal{E}$. Then $\left|\bar{a}_{i j}\right|<1$. We have $\bar{A} e=0, \pi \bar{A}=0$. That is, the Markov process with infinitesimal generator $\bar{A}$ has the same steadystate probability as the Markov process with infinitesimal generator $A$. Therefore, without loss of generality, we can assume that $A$ is normalized, i.e., $\left|a_{i j}\right| \leq 1$ for all $i, j \in \mathcal{E}$.

Let $f: \mathcal{E} \rightarrow \mathcal{R}$, where $\mathcal{R}=(-\infty, \infty)$ represents the space of real numbers. $f$ is called a performance function. The performance measure of the Markov process is defined as its expected value

$$
\eta=E_{\pi}(f)=\sum_{i \in \mathcal{E}} \pi_{i} f(i)=\pi f
$$

where $f=(f(1), f(2), \cdots)^{\prime}$ is a column vector, and $E_{\pi}$ denotes the expectation with respect to the steady-state measure $\pi$. (We use the same notation $f$ for both a function and a vector, with the $i$ th component $f_{i}=f(i), i \in \mathcal{E}$.)

Now suppose that $A$ changes to $A_{\delta}=\left[a_{\delta, i j}\right]=A+\delta Q$, with $\delta>0$ being a small real number, $Q=\left[q_{i j}\right]$, and $Q e=0$. Let $X_{\delta}$ be the Markov process with infinitesimal generator $A_{\delta}$. For $A_{\delta}$ to be an infinitesimal generator we require that $q_{i j} \geq 0$, if $a_{i j}=0, i \neq j$. It is clear that with this requirement $X_{\delta}$ is also irreducible. Let $\pi_{\delta}=\left(\pi_{\delta, 1}, \pi_{\delta, 2}, \cdots\right)$ be the vector of steady-state probabilities of $X_{\delta}$.

The performance measure of $X_{\delta}$ is $\eta_{\delta}=\eta+\Delta \eta$. We shall study the derivative of $\eta$ in the direction of $Q$, defined as

$$
\frac{\partial \eta}{\partial Q}=\lim _{\delta \rightarrow 0} \frac{\eta_{\delta}-\eta}{\delta} .
$$

Similar definitions will be used for other quantities, e.g., $\partial \pi / \partial Q=\lim _{\delta \rightarrow 0}\left(\pi_{\delta}-\pi / \delta\right)$ and $\partial A / \partial Q=\lim _{\delta \rightarrow 0}\left(A_{\delta}-\right.$ $A / \delta)=Q$. 


\section{THE REALIZATION MATRIX}

The concept of perturbation realization plays an important role in sensitivity analysis. The essential idea is that the effect of a parameter change can be decomposed into a sum of the effects of many individual changes on a sample path; the average effect of each individual change can be measured by a quantity called the realization factor. To apply this approach to study the sensitivity of a performance measure with respect to the infinitesimal generator, we first study the effect of the system changing from one state to another.

Recall that $X=\left\{X_{t}, t \geq 0\right\}$ is a Markov process with infinitesimal generator $A$ and state space $\mathcal{E}$. Let $\tilde{X}=\left\{\tilde{X}_{t}, t \geq\right.$ $0\}$ be another Markov process, independent of $X$, with the same state space $\mathcal{E}$ and the same infinitesimal generator $A$. The initial states of these two processes are, however, different. Denote them by $X_{0}=i, \tilde{X}_{0}=j, i, j \in \mathcal{E}$. Define $Y_{t}=$ $\left(X_{t}, \tilde{X}_{t}\right), t \geq 0$. Then $Y=\left\{Y_{t}, t \geq 0\right\}$ is a Markov process with state space $\mathcal{E} \times \mathcal{E}$.

We use superscripts $\{i\},\{j\}$, and $\{i, j\}$ to indicate the initial states of the processes. Thus, the three Markov processes are denoted as $X^{\{i\}}, \tilde{X}^{\{j\}}$, and $Y^{\{i, j\}}$, respectively. We use the same superscripts to represent quantities associated with these Markov processes. Thus, $\mathcal{F}_{t}^{\{i\}}=\sigma\left\{X_{s}^{\{i\}}, 0 \leq s \leq t\right\}, \mathcal{F}_{t}^{\{j\}}=$ $\sigma\left\{\tilde{X}_{s}^{\{j\}}, 0 \leq s \leq t\right\}$, and $\mathcal{F}_{t}^{\{i, j\}}=\sigma\left\{Y_{s}^{\{i, j\}}, 0 \leq s \leq t\right\}$. For any $0 \leq t \leq \infty, \mathcal{F}_{t}^{\{i, j\}}=\sigma\left\{\mathcal{F}_{t}^{\{i\}}, \mathcal{F}_{t}^{\{j\}}\right\}$, i.e., $\mathcal{F}_{t}^{\{\overline{i, j}\}}$ is the $\sigma$-field generated by $\mathcal{F}_{t}^{\{i\}}$ and $\mathcal{F}_{t}^{\{j\}}$. Furthermore, $\mathcal{F}_{\infty}^{\{i\}}$ and $\mathcal{F}_{\infty}^{\{j\}}$ are independent. Let $E$ be the expectations with respect to $\mathcal{F}_{\infty}^{\{i, j\}}$, and $p$ be the probability measure on $\mathcal{F}_{\infty}^{\{i, j\}}$.

$Y^{\{i, j\}}$ is regular, irreducible, and positive recurrent since $X^{\{i\}}$ and $\tilde{X}^{\{j\}}$ are. Thus, the first passage time from any state to any other state has a finite mean. Let $\mathcal{K}=\{(k, k): k \in \mathcal{E}\}$. Define

$$
T^{\{i, j\}}=\inf \left\{t: t \geq 0, Y_{t}^{\{i, j\}} \in \mathcal{K}\right\}
$$

$T^{\{i, j\}}$ is a stopping time of $Y^{\{i, j\}}$. At $T^{\{i, j\}}, Y^{\{i, j\}}$ reaches $\mathcal{K}$ for the first time and $E\left[T^{\{i, j\}}\right]$ is finite. After $T^{\{i, j\}}$, the two processes $X$ and $\tilde{X}$ are still independent; however, because $X_{T_{\{i, j\}}}=\tilde{X}_{T^{\{i, j\}}}$, they will behave similarly probabilistically afterwards. $T^{\{i, j\}}$ is just the coupling time of the two independent Markov processes with different initial distributions. Readers are referred to [38] for a survey of the relevant results about coupling.

Throughout the paper, we assume that $f$ is absolutely integrable, i.e.,

$$
E_{\pi}(|f|)=\sum_{i \in \mathcal{E}} \pi_{i}|f(i)|<\infty .
$$

With this assumption and some minor conditions, we can prove (see Appendix A)

$$
\lim _{t \rightarrow \infty} E\left[\left|f\left(X_{t}^{\{i\}}\right)\right|\right]=E_{\pi}(|f|), \quad i \in \mathcal{E} .
$$

Because $E\left[\left|f\left(X_{t}^{\{i\}}\right)\right|\right]$ is a continuous function of $t$ on $[0, \infty)$ (see, e.g., [15, eq. 8.4.8]), from (2) and (3), $E\left[\left|f\left(X_{t}^{\{i\}}\right)\right|\right]$ is bounded in $t \in[0, \infty)$.
Definition 1: $d_{i j}=E\left\{\int_{0}^{T^{\{i, j\}}}\left[f\left(\tilde{X}_{t}^{\{j\}}\right)-f\left(X_{t}^{\{i\}}\right)\right] d t\right\}, i$, $j \in \mathcal{E}$ is called a perturbation realization factor; the matrix $D=\left[d_{i j}\right]$ is called a realization matrix.

It is proved in Appendix $\mathrm{C}$ that $d_{i, j}<\infty$ for all $i, j \in \mathcal{E}$ (this can also be proved by using the concept of $f$-regularity defined in [37] and [38]). From the definition, we have

$$
d_{i j}=-d_{j i}, \quad i, j \in \mathcal{E}
$$

or equivalently, $D$ is skew-symmetric

$$
D^{\prime}=-D
$$

$d_{i j}$ measures the effect of a change from state $i$ to state $j$ on the performance of the system. In the terminology of perturbation analysis, we say that at $t=0$ the system state is perturbed from $i$ to $j$, and at $t=T^{\{i, j\}}$, the perturbation is realized by the system (i.e., the perturbed sample path returns back to the original one). This is similar to the realization of infinitesimal perturbations in discrete-event systems [7]. $d_{i j}$ can be written in a more convenient form as is given in the following theorem.

Theorem 1: If $E_{\pi}(|f|)<\infty$, then we have

$$
\begin{aligned}
d_{i j}=\lim _{T \rightarrow \infty}\left\{E\left[\int_{0}^{T} f\left(\tilde{X}_{t}^{\{j\}}\right) d t\right]-E\left[\int_{0}^{T} f\left(X_{t}^{\{i\}}\right) d t\right]\right\} \\
i, j \in \mathcal{E} .
\end{aligned}
$$

From the point of view of coupling, the result is very obvious. We give an intuitive explanation. First we have

$$
\begin{aligned}
\int_{0}^{\infty}\left[f\left(\tilde{X}_{t}^{\{j\}}\right)-f\left(X_{t}^{\{i\}}\right)\right] d t \\
=\int_{0}^{T^{\{i, j\}}}\left[f\left(\tilde{X}_{t}^{\{j\}}\right)-f\left(X_{t}^{\{i\}}\right)\right] d t \\
\quad+\int_{T^{\{i, j\}}}^{\infty}\left[f\left(\tilde{X}_{t}^{\{j\}}\right)-f\left(X_{t}^{\{i\}}\right)\right] d t .
\end{aligned}
$$

Next, because $\tilde{X}_{T\{i, j\}}^{\{j\}}=X_{T\{i, j\}}^{\{i\}}$, by the Markov property, we have $E\left\{\int_{T^{i, j\}}}^{\infty}\left[f\left(\tilde{X}_{t}^{\{j\}}\right)-f\left(X_{t}^{\{i\}}\right)\right] d t\right\}=0$. Thus, $E\left\{\int_{0}^{\infty}\left[f\left(\tilde{X}_{t}^{\{j\}}\right)-f\left(X_{t}^{\{i\}}\right)\right] d t\right\}=E\left\{\int_{0}^{T^{\{i, j\}}}\left[f\left(\tilde{X}_{t}^{\{j\}}\right)-\right.\right.$ $\left.\left.f\left(X_{t}^{\{i\}}\right)\right] d t\right\}$. Since $\int_{0}^{\infty}$ means $\lim _{T \rightarrow \infty} \int_{0}^{T}$, compared with the equation in Theorem 1, we need to prove that the order of "E" and $\lim _{T \rightarrow \infty}$ are exchangeable. A rigorous proof is provided in Appendix D.

To estimate $d_{i j}$ 's by using two independent sample paths is not convenient. The next theorem provides an easier approach for estimating $d_{i j}$ 's based on a single sample path. We define the first passage time from state $j$ to state $i, S^{\{j\}}(i)$, as follows:

$$
S^{\{j\}}(i)=\inf \left\{t: t \geq 0, \tilde{X}_{t}^{\{j\}}=i\right\} .
$$

$S^{\{j\}}(i)$ is a stopping time of $\tilde{X}^{\{j\}}$ and is independent of $X^{\{i\}}$. 
Theorem 2: If $E_{\pi}(|f|)<\infty$ and condition (3) holds, we have

$$
\begin{aligned}
d_{i j} & =E\left[\int_{0}^{S^{\{j\}}(i)} f\left(\tilde{X}_{t}^{\{j\}}\right) d t\right]-E\left[S^{\{j\}}(i)\right] \eta \\
& =E\left\{\int_{0}^{S^{\{j\}}(i)}\left[f\left(\tilde{X}_{t}^{\{j\}}\right)-\eta\right] d t\right\}, \quad i, j \in \mathcal{E} .
\end{aligned}
$$

The proof involves many technical details so we again put it in the Appendix. It is well known that the state probability distribution at $t$ converges to $\pi$ as $t$ goes to infinity. The condition required in the theorem is equivalent to $f\left(X_{t}^{\{i\}}\right)$ being uniformly integrable. In particular, this condition holds when $f$ is bounded.

Equation (5) has an interesting explanation: the mean effect of a state change from $i$ to $j$ on the performance measure (the realization factor $d_{i j}$ ) equals the mean of the accumulation of the deviation of $f\left(\tilde{X}_{t}^{\{j\}}\right)$ from its mean $\eta$ in the transition period from state $j$ to state $i$. From (5) and (4), we have

$$
\begin{aligned}
& E\left\{\int_{0}^{S^{\{j\}}(i)}\left[f\left(\tilde{X}_{t}^{\{j\}}\right)-\eta\right] d t\right\} \\
& =-E\left\{\int_{0}^{S^{\{i\}}(j)}\left[f\left(X_{t}^{\{i\}}\right)-\eta\right] d t\right\} .
\end{aligned}
$$

From Theorem 1, we have

$$
d_{i j}=d_{i k}+d_{k j}, \quad i, j, k \in \mathcal{E} .
$$

This property is similar to that of the potential energy in physics. In view of this, we may pick up any integer $k^{*} \in \mathcal{E}$ and any real number $c$ and define a quantity, $g_{i}$, called the performance potential, or simply the potential, of state $i$, as follows:

$$
g_{k^{*}}=c \quad \text { and } \quad g_{i}=g_{k^{*}}+d_{k^{*}}, \quad i \neq k^{*} .
$$

From (6), we can prove

$$
d_{i j}=g_{j}-g_{i}, \quad \text { for all } i, j \in \mathcal{E}
$$

i.e., a realization factor is the difference of two potentials. More about potentials will be discussed in the next subsection. Now we have

$$
D=e g^{\prime}-g e^{\prime}
$$

where $g=\left(g_{1}, g_{2}, \cdots\right)^{\prime}$ is called a potential vector. Let

$$
\mathcal{V}=\left\{e x^{\prime}-x e^{\prime}: x \in \mathcal{R}^{\infty}\right\} .
$$

Theorem 3: Suppose $E_{\pi}(|f|)<\infty$ and (3) holds. The realization matrix $D$ satisfies the Lyapunov equation

$$
A D+D A^{\prime}=-F
$$

where $F=e f^{\prime}-f e^{\prime}$. The solution is unique in $\mathcal{V}$, if the Markov process is strong ergodic.
Proof: Let $p_{i j}(t)=P\left\{X_{t}^{\{i\}}=j\right\}$ and $P(t)=$ $\left[p_{i j}(t)\right]_{i, j \in \mathcal{E}}$. Then we have $P(t)=\exp \{A t\}$ (see, e.g., [15]). Thus, $E\left[f\left(X_{t}^{\{i\}}\right)\right]=\Sigma_{k \in \mathcal{E}} f(k) p_{i k}(t)$, which is the $i$ th entry of $\exp \{A t\} f$. Let

$$
\begin{aligned}
d_{i j}(T) & =E\left\{\int_{0}^{T}\left[f\left(\tilde{X}_{t}^{\{j\}}\right)-f\left(X_{t}^{\{i\}}\right)\right] d t\right\} \\
& =\int_{0}^{T}\left\{E\left[f\left(\tilde{X}_{t}^{\{j\}}\right)\right]-E\left[f\left(X_{t}^{\{i\}}\right)\right]\right\} d t
\end{aligned}
$$

and $D(T)=\left[d_{i j}(T)\right]$. Then from Theorem 1

$$
D=\lim _{T \rightarrow \infty} D(T) .
$$

The integrand on the right-hand side of (10) equals the difference between the $j$ th and $i$ th entries of $\exp \{A t\} f$. Therefore

$$
D(T)=\int_{0}^{T}\left(e f^{\prime}[\exp \{A t\}]^{\prime}-[\exp \{A t\}] f e^{\prime}\right) d t .
$$

Using $A e=0$, we obtain

$$
\begin{aligned}
A D(T) & +D(T) A^{\prime} \\
= & \int_{0}^{T}\left(e f^{\prime}[\exp \{A t\}]^{\prime} A^{\prime}-A[\exp \{A t\}] f e^{\prime}\right) d t \\
= & e f^{\prime}\left(\int_{0}^{T}[\exp \{A t\}] A d t\right)^{\prime} \\
& -\left(\int_{0}^{T}[\exp \{A t\}] A d t\right) f e^{\prime} \\
= & e f^{\prime}[\exp \{A T\}-\exp \{0\}]^{\prime} \\
& -[\exp \{A T\}-\exp \{0\}] f e^{\prime} .
\end{aligned}
$$

The last two terms are finite by (2) and (3); thus, all the terms in (11) are meaningful. Note that $\lim _{T \rightarrow \infty} p_{i j}(T)=\pi_{j}$, i.e., $\lim _{T \rightarrow \infty} \exp \{A T\}=e \pi, \exp \{0\}=I$, and $e f^{\prime}(e \pi)^{\prime}=$ $(\pi f) e e^{\prime}=e \pi f e^{\prime}$. Letting $T \rightarrow \infty$ in (11), we get

$$
A D+D A^{\prime}=f e^{\prime}-e f^{\prime}=-F .
$$

Next, we prove the uniqueness of the solution. Suppose that (9) has two matrix solutions, $D_{1}=e g_{1}^{\prime}-g_{1} e^{\prime}$ and $D_{2}=e g_{2}^{\prime}-g_{2} e^{\prime}$. Let $W=D_{1}-D_{2}=e w^{\prime}-w e^{\prime}$, with $w=g_{1}-g_{2}$. Then $A W+W A^{\prime}=0$. Because $A e=0$, we have $e w^{\prime} A^{\prime}-A w e^{\prime}=0$. If the Markov process is strong ergodic, then the group inverse of $A$ (see the next section), $A^{\#}$, exists [34]. Multiplying both sides of this equation on the left with $A^{\#}$, and using $A^{\#} A=I-e \pi$ and $A^{\#} e=0$, we have $(I-e \pi) w e^{\prime}=0$. Therefore

$$
w e^{\prime}=e \pi w e^{\prime}=(\pi w) e e^{\prime}
$$

where $\pi w$ is a constant. From this, we have $W=\left(w e^{\prime}\right)^{\prime}-$ $w e^{\prime}=0$, i.e., $D_{1}=D_{2}$. 


\section{Potentials and Performance Sensitivities}

We first briefly review the concept of group inverse of a matrix. Since one eigenvalue of $A$ is zero, its regular inverse does not exist. We have to use a generalized inverse, i.e., the "group inverse" [36], which is related to the fundamental matrix $(A-e \pi)^{-1}$. Reference [33] proved that if $A$ is of a finite dimension, then its regular inverse exists. For matrices with an infinite dimension, we have a similar result. We state it as a lemma (for a proof, see [34, Th. 33]).

Lemma 1: If the Markov process is strong ergodic, then

$$
(A-e \pi)^{-1}=-\sum_{k=0}^{\infty}(P-e \pi)^{k}
$$

where $P=I+A$.

Since we assume that the Markov process is normalized, i.e., $\left|a_{i j}\right| \leq 1, a_{i j} \geq 0, i \neq j, a_{i i}<0$ for all $i, j \in \mathcal{E}$, we have $0 \leq$ $p_{i j} \leq 1$, for all $i, j \in \mathcal{E}$. The matrix $A^{\#}=(A-e \pi)^{-1}-e \pi$ is called the group inverse of $A$ [36]. In this paper, whenever $A^{\#}$ appears, we always assume that the Markov process is strong ergodic. It is straightforward to prove

$$
A A^{\#}=A^{\#} A=I-e \pi \text {. }
$$

Theorem 4: The derivative of the steady-state probability is

$$
\frac{\partial \pi}{\partial Q}=-\pi Q A^{\#}
$$

Furthermore, if $(\partial \pi / \partial Q) f=(\partial / \partial Q)(\pi f)$ and the results of all operators are finite, then the performance derivative can be calculated by using the group inverse of $A, A^{\#}$, or the realization matrix $D$, or the potential vector $g$

$$
\begin{aligned}
\frac{\partial \eta}{\partial Q} & =-\pi Q A^{\#} f \\
& =\pi Q D^{\prime} \pi^{\prime} \\
& =\pi Q g .
\end{aligned}
$$
we get

Proof: From $\pi_{\delta} A_{\delta}=0, A_{\delta}=A+\delta Q$, and $\pi A=0$,

$$
\frac{\pi_{\delta}-\pi}{\delta} A_{\delta}=-\pi Q
$$

Multiplying both sides on the right with $A_{\delta}^{\#}$ and using $A_{\delta} A_{\delta}^{\#}=\left(I-e \pi_{\delta}\right), \pi e=\pi_{\delta} e=1$, we get

$$
\frac{\pi_{\delta}-\pi}{\delta}=-\pi Q A_{\delta}^{\#}
$$

In the above equation, $Q$ is bounded and $\Sigma_{i} \pi_{i}$ converges. Letting $\delta \rightarrow 0$, we get

$$
\frac{\partial \pi}{\partial Q}=-\pi Q \lim _{\delta \rightarrow 0} A_{\delta}^{\#} .
$$

Now we prove that $A_{\delta}^{\#}$ is continuous, i.e., $\lim _{\delta \rightarrow 0} A_{\delta}^{\#}=A^{\#}$. It is equivalent to show

$$
\lim _{\delta \rightarrow 0}\left(A_{\delta}-e \pi_{\delta}\right)^{-1}=(A-e \pi)^{-1} .
$$

Consider a Banach space of all bounded real sequences $\mathcal{U}=\left\{u=\left(u_{1}, u_{2}, \cdots\right) ; u_{i} \in \mathcal{R}, i=1,2, \cdots\right\}$. The norm of $u$ is defined as $\|u\|=\sup _{i>1}\left|u_{i}\right|$. The norm of an operator $\mathcal{T}$ on $\mathcal{U}$ is defined as $\|\mathcal{T}\|=\sup _{\|u\|=1}\|\mathcal{T} u\|$. Any infinite dimensional matrix $R=\left[r_{i j}\right]_{i, j \in \mathcal{E}}$ can be considered as an operator on $\mathcal{U}$ with norm $\|R\|=\sup _{i} \sum_{j=1}^{\infty}\left|r_{i j}\right|$.

Let $x$ be any point in $\mathcal{U}$, and $(A-e \pi)^{-1} x=y$ and $\left(A_{\delta}-\right.$ $\left.e \pi_{\delta}\right)^{-1} x=y_{\delta}$. Then, $x=(A-e \pi) y$ and $x=\left(A_{\delta}-e \pi_{\delta}\right) y_{\delta}$. We have $y-y_{\delta}=(A-e \pi)^{-1}\left[\delta Q y_{\delta}+e\left(\pi-\pi_{\delta}\right) y_{\delta}\right]$. Note that $Q, y_{\delta}$, and $(A-e \pi)^{-1}$ are bounded (see [34] for the boundedness of $(A-e \pi)^{-1}$. It can also be proved by observing that $(A-e \pi)$ is a linear, bounded, continuous, and one-to-one onto mapping and applying the Banach theorem [43]). Thus, we have $\lim _{\delta \rightarrow 0} y_{\delta}=y$. This holds for any point in the Banach space; thus (20) follows. From (19), we obtain

$$
\frac{\partial \pi}{\partial Q}=-\pi Q A^{\#}
$$

Equation (15) follows directly from (14) and the assumption $(\partial \pi / \partial Q) f=(\partial / \partial Q)(\pi f)$.

Now we prove

$$
A^{\#} f=D \pi^{\prime}
$$

First, multiplying both sides of (9) on the right with $\pi^{\prime}$ and using $\pi A=0$ and $\pi e=1$, we have

$$
\begin{aligned}
A D \pi^{\prime} & =f e^{\prime} \pi^{\prime}-e f^{\prime} \pi^{\prime}=f-\pi f e \\
& =(I-e \pi) f .
\end{aligned}
$$

That is, $A D \pi^{\prime}=A A^{\#} f$. Multiplying both sides on the left with $A^{\#}$, we get $(I-e \pi) D \pi^{\prime}=(I-e \pi) A^{\#} f$. This directly leads to (21), because $\pi A^{\#}=0$ and

$\pi D \pi^{\prime}=\pi\left(e g^{\prime}-g e^{\prime}\right) \pi^{\prime}=g^{\prime} \pi^{\prime}-\pi g=0, \quad$ for all $D \in \mathcal{V}$

Finally, substituting (8) into (16) and using $Q e=0$ and $\pi e=1$, we obtain (17).

Remarks:

1) If the Markov process is finite, (14) can be simply proved by differentiating $\pi A=0$ in the direction of $Q$. Reference [44] studied the sensitivity problem of finite Markov chains with discrete time by using the inverse of the fundamental matrix.

2) If $\sup _{i \in \mathcal{E}}\left|f_{i}\right|<\infty$, in particular if the Markov process is finite, then multiplying both sides of (18) on the right by $f$ and letting $\delta \rightarrow 0$ immediately leads to $(\partial \pi / \partial Q) f=(\partial / \partial Q)(\pi f)$, and other assumptions are not needed.

3) The supremum norm is used in the proof of Theorem 4. If we assume that $A_{\delta}^{\#}$ is continuous in a stronger norm, i.e., the $f$-norm defined in [38], then the interchangeability condition used in the theorem, $(\partial \pi / \partial Q) f=$ $(\partial / \partial Q)(\pi f)$, can be relaxed. See Appendix B for details. However, it seems difficult to determine which of these two conditions is weaker. 
Theorem 4 has an intuitive explanation by using the concept of realization. To see this, let us consider a simplified case where $q_{l s}=-1, q_{l t}=1$, and $q_{i j}=0$ for $i \neq l$ or $j \neq t, s$. For this case, (16) becomes

$$
\begin{aligned}
\frac{\partial \eta}{\partial Q} & =\pi_{l} \sum_{j=1}^{M} \sum_{k=1}^{M} \pi_{j} q_{l k} d_{j k} \\
& =\pi_{l} \sum_{j=1}^{M} \pi_{j}\left(q_{l s} d_{j s}+q_{l t} d_{j t}\right) \\
& =\pi_{l} \sum_{j=1}^{M} \pi_{j}\left(d_{j t}-d_{j s}\right) \\
& =\pi_{l} d_{s t} .
\end{aligned}
$$

Thus, $\partial \eta / \partial Q$ equals the expected value of $d_{s t}$.

Next, (22) can be derived using the following intuitive argument. Observe a sample path $\left\{X_{n} ; 0 \leq n<L\right\}$. Among the $L$ transitions on the sample path, the Markov chain $X$ visits state $l$, on the average, $L \pi_{l}$ times. Because the probability that a transition from $l$ to $s$ changes to a transition from $l$ to $t$ is $\delta$, on the sample path there are, on the average, $L \pi_{l} \delta$ times when state $s$ changes to state $t$. Let $F_{L}=\Sigma_{n=0}^{L-1} f\left(X_{n}\right)$. Each time the state changes from $s$ to $t, F_{L}$ changes, on the average, by the amount $d_{s t}$ (see Definition 1). In addition, because $\delta$ can be chosen arbitrarily small and $T^{\{s, t\}}$ is always finite, the probability of two changes from $s$ to $t$ occurring within $T^{\{s, t\}}$ state transitions is of order $\delta^{2}$ and is hence negligible. This implies that the effect of each change can be treated separately. Therefore, the total change of $\eta$ due to the change of $P$ is

Finally

$$
\Delta \eta=\frac{1}{L} \Delta F_{L}=\frac{1}{L}\left\{L \pi_{l} \delta d_{s t}\right\}=\pi_{l} \delta d_{s t} .
$$

$$
\frac{\partial \eta}{\partial Q}=\lim _{\delta \rightarrow 0} \frac{\eta^{\prime}-\eta}{\delta}=\pi_{l} d_{s t}
$$

which is the same as (22). Roughly speaking, this means that the performance derivative equals the product of the effect of one jump from state $s$ to $t$ and the probability of such a jump. This is exactly the same as the performance realization theory in IPA of queueing networks (see [7]). Thus, both IPA and non-IPA approaches can be explained by using the same concept.

As defined in the last section, $g_{i}$ is the performance potential of state $i$, and $g$ is called the potential vector. From the definition in (7), if $g$ is a potential vector, then for any constant $c, g+c e$ is also a potential vector. We say that $g+c e$ is equivalent to $g$. This is similar to the potential energy in physics, where any point can be picked up as a reference point, and the only important thing is the difference between the potentials of any two points. This feature is reflected in (17): the derivative $\partial \eta / \partial Q$ depends only on the differences of the components of $g$ because $Q e=0, g$ in (17) can be replaced by $g+c e$ with any constant $c$.

All performance potentials satisfy the following Poisson equation:

$$
A g=-f+\pi f e .
$$

This shows that the potential defined through realization factors is in fact the same as the one defined by the Poisson equation. In other words, our analysis reveals the connection between the classical potential theory and PA, in particular, the concept of the realization factor. There is a great deal of literature on the Poisson equation, and many results, such as the existence, the uniqueness (modulo an additive constant), and the finiteness, and many other properties of its solution exist (see, e.g., [15], [24], [27], [34], [35], and [37]). Especially, the concept of $f$-regularity was used in [24] to prove the uniqueness of the solution; [24] also gives an interpretation of the solution to the Poisson equation that is similar to (7).

From Theorem 1, we have

$$
\begin{aligned}
g_{j}-g_{i}=\lim _{T \rightarrow \infty}\left\{E\left[\int_{0}^{T} f\left(\tilde{X}_{t}^{\{j\}}\right) d t\right]\right. \\
\left.-E\left[\int_{0}^{T} f\left(X_{t}^{\{i\}}\right) d t\right]\right\} .
\end{aligned}
$$

This shows that although the values of $E\left[\int_{0}^{\infty} f\left(X_{t}^{\{i\}}\right) d t\right]$ are infinite, their differences are finite and can be used as realization factors. In particular, for any finite $T$

$$
g_{i}(T)=E\left[\int_{0}^{T} f\left(X_{t}^{\{i\}}\right) d t\right]
$$

can be used as estimates of the potentials. It is clear that for any potential vector $g$, we have

$$
g_{j}-g_{i}=\lim _{T \rightarrow \infty}\left\{g_{j}(T)-g_{i}(T)\right\} .
$$

Let $g(T)=\left(g_{1}(T), g_{2}(T), \cdots, g_{M}(T)\right)^{\prime}$. Then

$$
D=\lim _{T \rightarrow \infty}\left\{e[g(T)]^{\prime}-[g(T)] e^{\prime}\right\} .
$$

To relate $E\left[\int_{0}^{T} f\left(X_{t}^{\{i\}}\right) d t\right]$ to $g=-A^{\#} f$, we need the following lemma.

Lemma 2:

$$
\begin{aligned}
A^{\#} & =-\int_{0}^{\infty}(\exp \{A t\}-e \pi) d t \\
& =-\lim _{T \rightarrow \infty}\left\{\int_{0}^{T} \exp \{A t\} d t-T e \pi\right\} .
\end{aligned}
$$

Proof: First, we have

$$
\int_{0}^{\infty} \exp \{A t\} A d t=-(I-e \pi) .
$$

From this, using $A e=\pi A=0$, we get

$$
\begin{aligned}
& A\left[\int_{0}^{\infty}(\exp \{A t\}-e \pi) d t\right] \\
& \quad=\left[\int_{0}^{\infty}(\exp \{A t\}-e \pi) d t\right] A=-(I-e \pi) .
\end{aligned}
$$

Furthermore, we can easily prove that

$$
\begin{aligned}
\pi\left[\int_{0}^{\infty}(\exp \{A t\}-e \pi) d t\right] \\
=\left[\int_{0}^{\infty}(\exp \{A t\}-e \pi) d t\right] e=0 .
\end{aligned}
$$


Multiplying both sides of (27) on the left with $A^{\#}$, we obtain (26).

Let $\epsilon^{k}(n)=1$ if $n=k$ and $\epsilon^{k}(n)=0$ if $n \neq k$. Then from (26), the $(i, k)$ th entry of $A^{\#}$ is

$$
\begin{aligned}
a_{i k}^{\#} & =-\lim _{T \rightarrow \infty}\left\{\int_{0}^{T} p_{i k}(t) d t-T \pi_{k}\right\} \\
& =-\lim _{T \rightarrow \infty}\left\{E\left[\int_{0}^{T} \epsilon^{k}\left(X_{t}^{\{i\}}\right) d t\right]-T \pi_{k}\right\} .
\end{aligned}
$$

The potentials are

$$
\begin{aligned}
g_{i} & =-\sum_{k \in \mathcal{E}} a_{i k}^{\#} f_{k} \\
& =\lim _{T \rightarrow \infty}\left\{E\left[\int_{0}^{T} f\left(X_{t}^{\{i\}}\right) d t\right]-T \eta\right\} .
\end{aligned}
$$

In modern Markov theory [15], the $\alpha$ potential of a function $f$ is defined as

$$
g_{i}^{(f)}=E\left[\int_{0}^{\infty} \exp \{-\alpha t\} f\left(X_{t}^{\{i\}}\right) d t\right] .
$$

Interestingly, our definition of potential is consistent with this classic definition with $\alpha=0$. Our results about realization and sensitivity analysis provide a new explanation and application for the Markov potential theory with $\alpha=0$.

\section{MARKOV CHAINS}

The results about Markov processes can be easily translated into those for Markov chains. Consider an irreducible and aperiodic Markov chain $X^{\{i\}}=\left\{X_{n}^{\{i\}} ; n \geq 0\right\}$ on a state space $\mathcal{E}=\{1,2, \cdots\}$ with transition probability matrix $P=$ $\left[p_{i j}\right]_{i, j \in \mathcal{E}}, P e=e$, and initial state $X_{0}=i$. This Markov chain can be considered as a uniformized embedded Markov chain in the Markov process $\left\{X_{t}^{\{i\}} ; t \geq 0\right\}$ with infinitesimal generator $A=P-I, A e=0$ [32].

Now we assume that $P$ changes to $P+\delta Q$, with $\delta>0$ being a small real number and $Q e=0$. We wish to estimate the derivative of $\eta$ in the direction of $Q, \partial \eta / \partial Q=$ $\lim _{\delta \rightarrow 0}(\Delta \eta / \delta)$. Because $A=P-I$, the change in $A$ is also $\delta Q$. Therefore, the results in the previous sections can be applied, with the same notations, to the corresponding Markov process with infinitesimal generator $A$. These results translate directly to the discrete version for the Markov chain with transition matrix $P$. We list some of them for references

$$
\begin{aligned}
d_{i j} & =\lim _{n \rightarrow \infty}\left\{E\left[\sum_{k=0}^{n} f\left(\tilde{X}_{k}^{\{j\}}\right)\right]-E\left[\sum_{k=0}^{n} f\left(X_{k}^{\{i\}}\right)\right]\right\} \\
& =E\left\{\sum_{k=0}^{L^{\{j\}}(i)-1}\left[f\left(\tilde{X}_{k}^{\{j\}}\right)-\eta\right]\right\}
\end{aligned}
$$

where $L^{\{j\}}(i)=\min \left\{n: n \geq 0, \tilde{X}_{n}^{\{j\}}=i\right\}$. The realization matrix $D=\left[d_{i j}\right]$ satisfies the discrete-time Lyapunov equation

$$
-D+P D P^{\prime}=-F \text {. }
$$

In addition

$$
\begin{aligned}
& A^{\#}=\lim _{n \rightarrow \infty}\left\{\sum_{k=0}^{n}\left(e \pi-P^{k}\right)\right\} \\
& a_{i k}^{\#}=-\lim _{n \rightarrow \infty}\left\{E\left[\sum_{l=0}^{n-1} \epsilon^{k}\left(X_{l}^{\{i\}}\right)\right]-n \pi_{k}\right\}
\end{aligned}
$$

and

$$
g_{i}=\lim _{n \rightarrow \infty}\left\{E\left[\sum_{l=0}^{n-1} f\left(X_{l}^{\{i\}}\right)\right]-n \eta\right\}
$$

\section{DISCUSSION}

We proposed two fundamental concepts, realization factors and performance potentials, for a Markov process. We showed that they can be determined by the group inverse of the infinitesimal generator of the Markov process and that the potentials defined through realization factors are solutions to the Poisson equation. The realization factors and the performance potentials can be estimated by analyzing a single sample path of a Markov process, and the sensitivity of the steady-state performance with respect to the change of the infinitesimal generator can be easily calculated by using either of these quantities.

Based on these results, some algorithms for estimating performance sensitivities on a single sample path of a Markov process can be proposed; these algorithms are simple, accurate, and widely applicable. The algorithms and simulation results will be presented in a separate paper. The results in this paper established a theoretical foundation for these practical algorithms.

The results provide a uniform framework for IPA and non-IPA approaches to the sensitivity analysis of steady-state performance; the central piece of this framework is the concept of perturbation realization, which, in the case of Markov processes, is closely related to potentials. Note that in [23] the Poisson equation for potentials was used to establish the uniqueness of the equations for realization factors in queueing networks; the problem is different from the one addressed in this paper.

The concept of potentials creates a new research direction in the area of single sample path based performance sensitivity analysis. The potential of a state is a measure of the average long-term performance of a Markov process starting from that state. It is therefore natural to use potentials in Markov decision problems where an action has to be taken at any state so that a long term performance will be optimized. This is an ongoing research topic.

\section{APPENDIX}

\section{A. The Proof of (3)}

We first review the concept of $f$-norm defined in [38]. Let $\nu$ be any signed measure on state space $\mathcal{E}$. We first assume $f \geq 1$ so that the results in [38] can be applied. Thus, $f: \mathcal{E} \rightarrow[1, \infty)$ is an arbitrary function. For countable state space, we can 
define a row vector, also denoted as $\nu$, such that $\nu_{i}$ is the measure of state $i$. Let $g$ denote a column vector. Then, in our notation, the $f$-norm of $\nu$ is defined as

$$
\|\nu\|_{f}=\sup _{g:|g| \leq f}|\nu g|
$$

Let $A$ be any matrix whose components are all uniformly bounded. $A$ maps any signed measure $\nu$ to anther signed measure $\nu A$. Thus, $A$ is an operator defined on the linear space of the signed measures. The induced $f$-norm of $A$ is defined as

$$
\begin{aligned}
\|A\|_{f} & =\sup _{\nu: \nu f<\infty} \frac{\|\nu A\|_{f}}{\|\nu\|_{f}} \\
& =\sup _{\nu: \nu f<\infty} \frac{\sup _{g:|g| \leq f}\{|\nu A g|\}}{\sup _{g:|g| \leq f}\{|\nu g|\}} .
\end{aligned}
$$

Next, let $I_{i}$ be a unit row vector with its $i$ th component being one and all the other components being zero. We have

$$
\begin{aligned}
I_{i} A f & \leq \sup _{g:|g| \leq f}\left\{\left|I_{i} A g\right|\right\} \\
& =\left(\frac{\sup _{g:|g| \leq f}\left\{\left|I_{i} A g\right|\right\}}{\sup _{g:|g| \leq f}\left\{\left|I_{i} g\right|\right\}}\right) \sup _{g:|g| \leq f}\left\{\left|I_{i} g\right|\right\} \\
& \leq\left(\sup _{\nu: \nu f<\infty} \frac{\sup _{g:|g| \leq f}\{|\nu A g|\}}{\sup _{g:|g| \leq f}\{|\nu g|\}}\right) \sup _{g:|g| \leq f}\left\{\left|I_{i} g\right|\right\} \\
& =\|A\|_{f} \sup _{g:|g| \leq f}\left\{\left|I_{i} g\right|\right\} \\
& \leq\|A\|_{f} f_{i} .
\end{aligned}
$$

Writing it in a vector form, we have

$$
A f \leq\|A\|_{f} f
$$

From (31), we can easily prove

$$
A^{k} f \leq\|A\|_{f}^{k} f, \quad k=1,2, \cdots
$$

and

$$
\{\exp (A)\} f \leq \exp \left\{\|A\|_{f}\right\} f .
$$

Recall that $p_{i j}(t)=P\left\{X_{t}^{\{i\}}=j\right\}$ and $P(t)=\left[p_{i j}(t)\right]_{i, j \in \mathcal{E}}$ $=\exp \{A t\}$, and we have

$$
P(t) f \leq \exp \left\{\|A\|_{f} t\right\} f .
$$

Now we assume that $\|A\|_{f}$ is finite. Thus, with (32) and $\pi f \leq \infty$, from [39, Th. 7.2], we conclude that the Markov chain is $f$-ergodic, i.e.,

$$
\lim _{t \rightarrow \infty}\left\|p_{i, \cdot}(t)-\pi\right\|_{f}=0, \quad \text { for all } i \in \mathcal{E} .
$$

In our notation, this is the same as (3).

If all the components of $|f|$ are uniformly bounded, then $\|A\|_{f}$ is finite. Another simple condition for $\|A\|_{f}$ to be finite is $|A| f \leq K f$, where $|A|=\left[\left|a_{i j}\right|\right]$ and $K$ is a constant. Since $A=P-I$, the above condition is equivalent to $P f \leq K f$ (recall that $f \geq 1$ ).

Finally, if $f \geq 1$ does not hold, we can set $f=f^{(1)}+f^{(2)}$ such that $\left|f^{(1)}\right| \geq 1$ and $\left|f^{(2)}\right| \leq 1$. (Suppose $f_{i}=\alpha$ and $|\alpha|<1$, then we can choose $f^{(1)}=\operatorname{sign}(\alpha)(1+|\alpha|)$ and $f^{(2)}=-\operatorname{sign}(\alpha)$.) We have proved that (3) holds $\left|f^{(1)}\right|$, and it obviously holds for $f^{(2)}$.

\section{B. Theorem 4 with f-Norm}

Any row in $A^{\#}$ can be considered as a measure on $\mathcal{E}$. Now we assume that every such measure is continuous in $f$-norm, i.e.,

$$
\lim _{\delta \rightarrow 0}\left\|A_{\delta, i, \cdot}^{\#}-A_{i, \cdot}^{\#}\right\|_{f}=0, \quad \text { for all } i .
$$

This implies

$$
\lim _{\delta \rightarrow 0}\left[A_{\delta, i, \cdot}^{\#}-A_{i, \cdot}^{\#}\right] f=0 .
$$

Thus, from (18), we obtain (15). That is, if for every row $A_{\delta}^{\#}$ is continuous in $f$-norm, then (15) holds.

Next, as in Appendix $\mathrm{A}, A_{\delta}^{\#}$ can also be viewed as an operator defined by $\nu A_{\delta}^{\#}$ and hence has an induced $f$-norm. Using the same argument as in Appendix A, we can prove that if $A_{\delta}^{\#}$ is continuous in this induced $f$-norm, then it is also continuous in the f-norm regarding every row, i.e., (33) holds. Thus (15) also holds.

Finally, the interchangeability condition $(\partial \pi / \partial Q) f=$ $(\partial / \partial Q)(\pi f)$ follows directly from (15) and (14).

\section{Some Boundedness Results}

With $E_{\pi}(|f|)<\infty$ and (3), we have

$$
E\left\{\int_{0}^{S^{\{j\}}(i)}\left|f\left(X_{t}^{\{i\}}\right)\right| d t\right\}<\infty .
$$

With $E_{\pi}(|f|)<\infty$, we have

$$
E\left\{\int_{0}^{S^{\{j\}}(i)}\left|f\left(\tilde{X}_{t}^{\{j\}}\right)\right| d t\right\}<\infty
$$

and

$$
E\left\{\int_{0}^{T^{\{i, j\}}}\left|f\left(X_{t}^{\{i\}}\right)\right| d t\right\}<\infty
$$

Proof:

1) First, with (3), $E\left[\mid f\left(X_{t}^{\{i\}}\right) \|\right]$ is bounded in $t \in[0, \infty)$. Next, by the independence of $S^{\{j\}}(i)$ and $X_{t}^{\{i\}}$, we have

$$
\begin{aligned}
& E\left\{\int_{0}^{S^{\{j\}}(i)}\left|f\left(X_{t}^{\{i\}}\right)\right| d t\right\} \\
& \quad=E\left\{\int_{0}^{S^{\{j\}}(i)} E\left[\left|f\left(X_{t}^{\{i\}}\right)\right|\right] d t\right\} \\
& <\sup _{t \geq 0}\left\{E\left[\left|f\left(X_{t}^{\{i\}}\right)\right|\right]\right\} E\left[S^{\{j\}}(i)\right]<\infty .
\end{aligned}
$$

2) Equation (34) is a direct consequence of the $f$-regularity defined in [37] and [38]. It is proved in [38] that an irreducible Markov chain is $f$-regular. Therefore, the resolvent chain (see [37]) is $f$-regular. From [37, Proposition 4.3], the $f$-regularity of a process is equivalent 
to the $f$-regularity of the resolvent chain. Thus, an irreducible Markov process is also $f$-regular. This leads to (34).

In the following, we provide an alternative proof that is based on the basic probability theory. For simplicity, we omit the tilde on $X$. Let $T_{k}, k=0,1,2, \cdots$, be the $k$ th transition epoch of $X$. Let $T_{0}=0, X_{0}=j, \tau_{0}=0$, and

$$
\begin{aligned}
v_{k} & =\min \left\{n \geq \tau_{k}, X_{T_{n}}=i\right\} \\
\tau_{k+1} & =\min \left\{n \geq v_{k}, X_{T_{n}}=j\right\} .
\end{aligned}
$$

That is, $T_{v_{k}}$ ( $T_{\tau_{k+1}}$, respectively) is the first time that $X$ transits to state $i$ ( $j$, respectively) after it transits to state $j$ ( $i$, respectively) at $T_{\tau_{k}}\left(T_{v_{k}}\right.$, respectively). From the regenerative property and ergodicity, we have

$$
\begin{aligned}
E\left\{\int_{0}^{S^{\{j\}}(i)}\left|f\left(X_{t}^{\{j\}}\right)\right| d t+\int_{0}^{S^{\{j\}}(j)}\left|f\left(X_{t}^{\{j\}}\right)\right| d t\right\} \\
=\lim _{L \rightarrow \infty} \frac{1}{L}\left\{\sum_{k=0}^{L-1} \int_{T_{\tau_{k}}}^{T_{v_{k}}}\left|f\left(X_{t}^{\{j\}}\right)\right| d t\right. \\
\left.\quad+\sum_{k=0}^{L-1} \int_{T_{v_{k}}}^{T_{\tau_{k+1}}}\left|f\left(X_{t}^{\{j\}}\right)\right| d t\right\} \\
=\lim _{L \rightarrow \infty} \frac{T_{\tau_{L}} \frac{1}{T_{\tau_{L}}} \int_{0}^{T_{\tau_{L}}}\left|f\left(X_{t}^{\{j\}}\right)\right| d t}{\sum_{k=0}^{L-1}\left(T_{v_{k}}-T_{\tau_{k}}\right)+\sum_{k=0}^{L}\left(T_{\tau_{k+1}}-T_{v_{k}}\right)} \\
=\lim _{L \rightarrow \infty} \frac{1}{L=0} \int_{\tau_{\tau_{L}}}^{T_{\tau_{L}}}\left|f\left(X_{t}^{\{j\}}\right)\right| d t \\
=\left\{E\left[S^{\{j\}}(i)\right]+E\left[S^{\{i\}}(j)\right]\right\} \pi|f| .
\end{aligned}
$$

In the above equation, for regular Markov processes, we have $\Sigma_{k=0}^{L-1}\left(T_{v_{k}}-T_{\tau_{k}}\right) \rightarrow \infty$ and $\Sigma_{k=0}^{L-1}\left(T_{\tau_{k+1}}-\right.$ $\left.T_{v_{k}}\right) \rightarrow \infty$ as $L \rightarrow \infty$. Furthermore, for positive recurrent Markov processes, $E\left[S^{\{j\}}(i)\right]<\infty$ and $E\left[S^{\{i\}}(j)\right\}<\infty$. Thus, from (37)

$$
E\left\{\int_{0}^{S^{\{j\}}(i)}\left|f\left(X_{t}^{\{j\}}\right)\right| d t\right\}<\infty .
$$

3) Consider $Y=\left\{\left(X_{t}, \tilde{X}_{t}\right), t \geq 0\right\}$. We apply the same notation as those in 2) for $X$; thus, $S^{\{i, j\}}(\{k, k\})$ is the first time that $Y$ starting from $\{i, j\}$ reaches $\{k, k\}$. Therefore

$$
T^{\{i, j\}}=\min \left\{S^{\{i, j\}}(\{k, k\}), k \in \mathcal{K}\right\}
$$

where $\mathcal{K}=\{(k, k) ; k \in \mathcal{E}\}$. Applying (35) to $Y$, we get $E\left\{\int^{S_{0}^{\{i, j\}}(\{k, k\})}\left|f\left(\tilde{X}_{t}^{\{j\}}\right)\right| d t\right\}<\infty, \quad k \in \mathcal{E}$

From (38) and (39), we get (36).

\section{The Proof of Theorem 1}

Let $I_{[V]}$ be the indicator function of event $V$. For any fixed value of $T$, we have

$$
\begin{aligned}
& E\left\{\int_{0}^{T}\left[f\left(\tilde{X}_{t}^{\{j\}}\right)-f\left(X_{t}^{\{i\}}\right)\right] d t\right\} \\
& =E\left\{\int_{0}^{T}\left[f\left(\tilde{X}_{t}^{\{j\}}\right)-f\left(X_{t}^{\{i\}}\right)\right] I_{\left[T^{\{i, j\}} \leq T\right]} d t\right\} \\
& +E\left\{\int_{0}^{T}\left[f\left(\tilde{X}_{t}^{\{j\}}\right)-f\left(X_{t}^{\{i\}}\right)\right] I_{\left[T^{\{i, j\}}>T\right]} d t\right\} \\
& =E\left\{[ \int _ { 0 } ^ { T ^ { \{ i , j \} } } + \int _ { T ^ { \{ i , j \} } } ^ { T } ] \left[f\left(\tilde{X}_{t}^{\{j\}}\right)\right.\right. \\
& \left.\left.-f\left(X_{t}^{\{i\}}\right)\right] I_{\left[T^{\{i, j\}} \leq T\right]} d t\right\} \\
& +E\left\{[ \int _ { 0 } ^ { T ^ { \{ i , j \} } } - \int _ { T } ^ { T ^ { \{ i , j \} } } ] \left[f\left(\tilde{X}_{t}^{\{j\}}\right)\right.\right. \\
& \left.\left.-f\left(X_{t}^{\{i\}}\right)\right] I_{\left[T^{\{i, j\}}>T\right]} d t\right\} \\
& =E\left\{\int_{0}^{T^{\{i, j\}}}\left[f\left(\tilde{X}_{t}^{\{j\}}\right)-f\left(X_{t}^{\{i\}}\right)\right] d t\right\} \\
& +E\left\{\int_{T^{\{i, j\}}}^{T}\left[f\left(\tilde{X}_{t}^{\{j\}}\right)-f\left(X_{t}^{\{i\}}\right)\right] I_{\left[T^{\{i, j\}} \leq T\right]} d t\right\} \\
& -E\left\{\int_{T}^{T^{\{i, j\}}}\left[f\left(\tilde{X}_{t}^{\{j\}}\right)-f\left(X_{t}^{\{i\}}\right)\right] I_{\left[T^{\{i, j\}}>T\right]} d t\right\} .
\end{aligned}
$$

For the second term of the most right-hand side of (40), we have

$$
\begin{aligned}
E\left\{\int_{T^{\{i, j\}}}^{T}\left[f\left(\tilde{X}_{t}^{\{j\}}\right)-f\left(X_{t}^{\{i\}}\right)\right] I_{\left[T^{\{i, j\}} \leq T\right]} d t\right\} \\
=E\left\{E \left\{\int _ { T ^ { \{ i , j \} } } ^ { T } \left[f\left(\tilde{X}_{t}^{\{j\}}\right)\right.\right.\right. \\
\left.\left.\left.-f\left(X_{t}^{\{i\}}\right)\right] I_{\left[T^{\{i, j\}} \leq T\right]} d t \mid \mathcal{F}_{T^{\{i, j\}}}^{\{i, j\}}\right\}\right\} .
\end{aligned}
$$

$T^{\{i, j\}}$ is measurable with respect to $\mathcal{F}_{T^{\{i, j\}}}^{\{i, j\}}$; thus

$$
\begin{gathered}
E\left\{\int_{T^{\{i, j\}}}^{T}\left[f\left(\tilde{X}_{t}^{\{j\}}\right)-f\left(X_{t}^{\{i\}}\right)\right] I_{\left[T^{\{i, j\}} \leq T\right]} d t \mid \mathcal{F}_{T^{\{i, j\}}}^{\{i, j\}}\right\} \\
=\int_{T^{\{i, j\}}}^{T} E\left\{\left[f\left(\tilde{X}_{t}^{\{j\}}\right)-f\left(X_{t}^{\{i\}}\right)\right] \mid \mathcal{F}_{T^{\{i, j\}}}^{\{i, j\}}\right\} \\
\cdot I_{\left[T^{\{i, j\}} \leq T\right]} d t .
\end{gathered}
$$

Note that $X_{T^{\{i, j\}}}=\tilde{X}_{T^{\{i, j\}}}$. Since $\tilde{X}^{\{j\}}$ and $X^{\{i\}}$ have the same infinitesimal generator, we have

$$
E\left\{\left[f\left(\tilde{X}_{t}^{\{j\}}\right)-f\left(X_{t}^{\{i\}}\right)\right] \mid \mathcal{F}_{T\{i, j\}}^{\{i, j\}}\right\}=0, \quad t \geq T^{\{i, j\}} .
$$


Therefore, the second term of the most right-hand side of (40) is zero. For the last term in (40), we have

$$
\begin{aligned}
& \left|\int_{T}^{T^{\{i, j\}}}\left[f\left(\tilde{X}_{t}^{\{j\}}\right)-f\left(X_{t}^{\{i\}}\right)\right] I_{\left[T^{\{i, j\}}>T\right]} d t\right| \\
& \quad<\int_{0}^{T^{\{i, j\}}}\left|f\left(\tilde{X}_{t}^{\{j\}}\right)\right| d t+\int_{0}^{T^{\{i, j\}}}\left|f\left(X_{t}^{\{i\}}\right)\right| d t .
\end{aligned}
$$

Note that $\lim _{T \rightarrow \infty} I_{\left[T^{\{i, j\}}>T\right]}=0$, w.p. 1. Thus, from (36) and by the dominated convergence theorem, the last term in (40) goes to zero as $T \rightarrow \infty$. Finally, taking $T \rightarrow \infty$, we get Theorem 1.

\section{E. The Proof of Theorem 2}

Using the notations in Section III, we have

$$
\begin{aligned}
& E\left\{\int_{0}^{T}\left[f\left(\tilde{X}_{t}^{\{j\}}\right)-f\left(X_{t}^{\{i\}}\right)\right] d t\right\} \\
& =E\left\{\int_{0}^{T}\left[f\left(\tilde{X}_{t}^{\{j\}}\right)-f\left(X_{t}^{\{i\}}\right)\right] I_{[S\{j\}(i) \leq T]} d t\right\} \\
& +E\left\{\int_{0}^{T}\left[f\left(\tilde{X}_{t}^{\{j\}}\right)-f\left(X_{t}^{\{i\}}\right)\right] I_{\left[S^{\{j\}}(i)>T\right]} d t\right\} \\
& =E\left\{\int_{0}^{S^{\{j\}}(i)} f\left(\tilde{X}_{t}^{\{j\}}\right) I_{[S\{j\}(i) \leq T]} d t\right\} \\
& -E\left\{\int_{T-S^{\{j\}(i)}}^{T} f\left(X_{t}^{\{i\}}\right) I_{\left[S^{\{j\}}(i) \leq T\right]} d t\right\} \\
& +E\left\{\int_{S^{\{j\}}(i)}^{T} f\left(\tilde{X}_{t}^{\{j\}}\right) I_{\left[S^{\{j\}}(i) \leq T\right]} d t\right\} \\
& -E\left\{\int_{0}^{T-S^{\{j\}}(i)} f\left(X_{t}^{\{i\}}\right) I_{[S\{j\}(i) \leq T]} d t\right\} \\
& +E\left\{\int_{0}^{S^{\{j\}}(i)} f\left(\tilde{X}_{t}^{\{j\}}\right) I_{[S\{j\}(i)>T]} d t\right\} \\
& -E\left\{\int_{0}^{T} f\left(X_{t}^{\{i\}}\right) I_{\left[S^{\{j\}}(i)>T\right]} d t\right\} \\
& -E\left\{\int_{T}^{S^{\{j\}}(i)} f\left(\tilde{X}_{t}^{\{j\}}\right) I_{\left[S^{\{j\}}(i)>T\right]} d t\right\} .
\end{aligned}
$$

For the last term of (41), we have

$$
\left.\mid \int_{T}^{S^{\{j\}}(i)} f\left(\tilde{X}_{t}^{\{j\}}\right) I_{\left[S^{\{j\}}(i)>T\right]} d t\right\}\left|<\int_{0}^{S^{\{j\}}(i)}\right| f\left(\tilde{X}_{t}^{\{j\}}\right) \mid d t
$$

which is integrable by (35). Note that $\lim _{T \rightarrow \infty} I_{[S\{j\}(i)>T]}=$ 0 . Then by the dominated convergence theorem, it is easy to prove that

$$
\lim _{T \rightarrow \infty} E\left\{\int_{T}^{S^{\{j\}}(i)} f\left(\tilde{X}_{t}^{\{j\}}\right) I_{\left[S^{\{j\}}(i)>T\right]} d t\right\}=0 .
$$

For the next to the last term in (41), we have

$$
\left|\int_{0}^{T} f\left(X_{t}^{\{i\}}\right) I_{[S\{j\}(i)>T]} d t\right| \leq \int_{0}^{S^{\{j\}}(i)}\left|f\left(X_{t}^{\{i\}}\right)\right| d t .
$$

Again, by (34) we can apply the dominated convergence theorem and obtain

$$
\lim _{T \rightarrow \infty} E\left\{\int_{0}^{T} f\left(X_{t}^{\{i\}}\right) I_{\left[S^{\{j\}}(i)>T\right]} d t\right\}=0
$$

Next, for the third term on the right-hand side of (41), we have

$$
\begin{aligned}
& E\left\{\int_{S^{\{j\}(i)}}^{T} f\left(\tilde{X}_{t}^{\{j\}}\right) I_{\left[S^{\{j\}}(i) \leq T\right]} d t\right\} \\
& \quad=E\left\{E\left[\int_{S^{\{j\}(i)}}^{T} f\left(\tilde{X}_{t}^{\{j\}}\right) I_{\left[S^{\{j\}}(i) \leq T\right]} d t \mid \mathcal{F}_{S^{\{j\}}(i)}^{\{j\}}\right]\right\} .
\end{aligned}
$$

Since $S^{\{j\}}(i)$ is measurable w.r.t. $\mathcal{F}_{S^{\{j\}(i)}}^{\{j\}}$, this equals

$$
\begin{gathered}
E\left\{\int_{S^{\{j\}(i)}}^{T} E\left[f\left(\tilde{X}_{t}^{\{j\}}\right) \mid \mathcal{F}_{S\{j\}(i)}^{\{j\}}\right] I_{[S\{j\}(i) \leq T]} d t\right\} \\
=E\left\{\int_{0}^{T-S^{\{j\}}(i)} E\left[f\left(\tilde{X}_{t+S\{j\}(i)}^{\{j\}}\right) \mid \mathcal{F}_{S^{\{j\}}(i)}^{\{j\}}\right]\right. \\
\left.\cdot I_{\left[S^{\{j\}}(i) \leq T\right]} d t\right\} .
\end{gathered}
$$

By the definition of $S^{(j)}(i)$, we have

$$
E\left[f\left(\tilde{X}_{t+S\{j\}(i)}^{\{j\}}\right) \mid \mathcal{F}_{S\{j\}(i)}^{\{j\}}\right]=E\left[f\left(\tilde{X}_{t}^{\{i\}}\right)\right]=E\left[f\left(X_{t}^{\{i\}}\right)\right] .
$$

Therefore, by the independence of $X^{\{i\}}$ and $S^{\{j\}}(i)$, we get

$$
\begin{aligned}
& E\left\{\int_{S^{\{j\}}(i)}^{T} f\left(\tilde{X}_{t}^{\{j\}}\right) I_{\left[S^{\{j\}}(i) \leq T\right]} d t\right\} \\
& =E\left\{\int_{0}^{T-S^{\{j\}}(i)} E\left[f\left(X_{t}^{\{i\}}\right)\right] I_{\left[S^{\{j\}}(i) \leq T\right]} d t\right\} \\
& =E\left\{\int_{0}^{T-S^{\{j\}}(i)} f\left(X_{t}^{\{i\}}\right) I_{\left[S^{\{j\}}(i) \leq T\right]} d t\right\} .
\end{aligned}
$$

Thus, the third and the fourth terms on the right-hand side of (41) cancel each other. Summarizing the above results by taking $T \rightarrow \infty$ on both sides of (41), we obtain

$$
\begin{aligned}
d_{i j}= & \lim _{T \rightarrow \infty} E\left\{\int_{0}^{T}\left[f\left(\tilde{X}_{t}^{\{j\}}\right)-f\left(X_{t}^{\{i\}}\right)\right] d t\right\} \\
=E & \left\{\int_{0}^{S^{\{j\}}(i)} f\left(\tilde{X}_{t}^{\{j\}}\right) d t\right\}-\lim _{T \rightarrow \infty} \\
& \cdot E\left\{\int_{T_{S}^{\{j\}}(i)}^{T} f\left(X_{t}^{\{i\}}\right) I_{\left[S^{\{j\}}(i) \leq T\right]} d t\right\} .
\end{aligned}
$$

By the independence of $X^{\{i\}}$ and $\tilde{X}^{\{j\}}$, the last term in (42) equals

$$
\lim _{T \rightarrow \infty} E\left\{\int_{T_{S}^{\{j\}}(i)}^{T}\left[E f\left(X_{t}^{\{i\}}\right)\right] I_{\left[S^{\{j\}}(i) \leq T\right]} d t\right\}
$$


in which $E\left[f\left(X_{t}^{\{i\}}\right)\right]=E\left[f\left(X_{t}^{\{i\}}\right) \mid \mathcal{F}_{S\{j\}(i)}^{\{j\}}\right]$. Next, we prove

$$
\begin{aligned}
\lim _{T \rightarrow \infty} & E\left\{\int_{T_{S}^{\{j\}}(i)}^{T}\left[E f\left(X_{t}^{\{i\}}\right)\right] I_{\left[S^{\{j\}}(i) \leq T\right]} d t\right\} \\
= & E\left[S^{\{j\}}(i)\right] \eta, \quad \text { w.p. } 1 .
\end{aligned}
$$

For any $T$, we first write

$$
S^{\{j\}}(i)=S^{\{j\}}(i) I_{\left[S^{\{j\}}(i) \leq T\right]}+S^{\{j\}}(i) I_{[S\{j\}(i)>T]} .
$$

Then we have

$$
\begin{aligned}
\left|E\left\{\int_{T_{S}^{\{j\}}{ }_{(i)}^{T}}^{T}\left[E f\left(X_{t}^{\{i\}}\right)\right] I_{\left[S^{\{j\}}(i) \leq T\right]} d t\right\}-E\left[S^{\{j\}}(i)\right] \eta\right| \\
=\mid E \int_{T_{S}^{\{j\}}(i)}^{T}\left\{E\left[f\left(X_{t}^{\{i\}}\right)\right]-\eta\right\} I_{\left[S^{\{j\}}(i) \leq T\right]} d t \\
\quad-E\left\{S^{\{j\}}(i) I_{\left[S^{\{j\}}(i)>T\right]}\right\} \eta \mid .
\end{aligned}
$$

By the dominated convergence theorem, the last term on the right-hand side tends to zero as $T \rightarrow \infty$. The first term on the right-hand side is dominated by

$$
E\left\{\max _{T-S^{\{j\}}(i) \leq t \leq T}\left|E\left[f\left(X_{t}^{\{i\}}\right)\right]-\eta\right| S^{\{j\}}(i) I_{\left[S^{\{j}(i) \leq T\right]}\right\} .
$$

Again, applying the dominated convergence theorem to (45) and using (37) we have

$$
\begin{aligned}
\lim _{T \rightarrow \infty} E\left\{\max _{T-S\{j\}(i) \leq t \leq T} \mid E\left[f\left(X_{t}^{\{i\}}\right)\right]\right. \\
\left.-\eta \mid S^{\{j\}}(i) I_{\left[S^{\{j\}}(i) \leq T\right]}\right\}=0 .
\end{aligned}
$$

Consequently, the first term also tends to zero as $T \rightarrow \infty$, again by the dominated convergence theorem. This proves (44). Putting (42)-(44) together yields (5).

\section{ACKNOWLEDGMENT}

The authors greatly appreciate the reviewers' comments, which have helped improve the quality of the paper. In particular, they are indebted to one reviewer for pointing out the relations of the potentials with the Poisson equation and suggesting the approach based on the $f$-norm.

\section{REFERENCES}

[1] P. Brémaud, "Maximal coupling and rare perturbation sensitivity analysis," Queueing Syst.: Theory Appl., vol. 10, pp. 249-270, 1992.

[2] C. A. Brooks and P. Varaiya, "Using perturbation analysis to solve the capacity and flow assignment problem for general and ATM networks," in Proc. IEEE Globcom, 1994.

[3] A. Berman and R. J. Plemmons, Nonnegative Matrices in the Mathematical Sciences. Philadelphia, PA: SIAM, 1994.

[4] L. Breiman, Probability. New York: Addison Wesley, 1968; also New York: Springer-Verlag, 1994

[5] X. R. Cao, "Convergence of parameter sensitivity estimates in a stochastic experiment," IEEE Trans. Automat. Contr., vol. AC-30, pp. 834-843, 1985.

[6] _ source queue," Performance Eval., vol. 7, pp. 31-41, 1987.
[7] _ Realization Probabilities: The Dynamics of Queueing Systems. New York: Springer-Verlag, 1994

[8] _ "Uniformization and performance sensitivity estimation in closed queueing networks," Math. Computer Modeling, vol. 23, no. 12/12, pp. 77-92, 1996

[9] X. R. Cao, X.-M. Yuan, and L. Qiu, "A new formula for performance sensitivities of discrete-time Markov chains," IEEE Trans. Automat. Contr., vol. 41, pp. 1814-1817, 1996

[10] X. R. Cao and Y.-W. Wan, "Algorithms for sensitivity analysis of Markov systems through potentials and perturbation realization," IEEE Trans. Contr. Syst. Technol., to be published.

[11] M. Caramanis and G. Liberopoulos, "Perturbation analysis for the design of flexible manufacturing system flow controllers," Operations Res., vol. 40, pp. 1107-1125, 1992.

[12] C. G. Cassandras, Discrete Event Systems: Modeling and Performance Analysis. Aksen, 1993.

[13] C. G. Cassandras and S. G. Strickland, "On-line sensitivity analysis of Markov chains," IEEE Trans. Automat. Contr., vol. 34, pp. 76-86, 1989.

[14] E. K. P. Chong and P. J. Ramadge, "Optimization of queues using infinitesimal perturbation analysis-based algorithms with general update times," SIAM J. Contr. Optim., vol. 31, pp. 698-732, 1993.

[15] E. Cinlar, Introduction to Stochastic Processes. Englewood Cliffs, NJ: Prentice-Hall, 1975.

[16] L. Y. Dai, "A consistent algorithm for derivative estimation of Markov chains," in Proc. 33rd IEEE Conf. Decision Contr., 1994, pp. 1990-1995.

[17] L. Y. Dai and Y. C. Ho, "Structural infinitesimal perturbation analysis (SIPA) for derivative estimation of discrete event dynamic systems," IEEE Trans. Automat. Contr., vol. 40, pp. 1154-1166, 1995.

[18] N. M. Van Dijk, "On a simple proof of uniformization for continuous and discrete-state continuous-time Markov chains," Advances Appl. Probability, vol. 22, pp. 749-750, 1990.

[19] M. C. Fu and J. Q. Hu, "Extensions and generalizations of smoothed perturbation analysis in a generalized semi-Markov process framework," IEEE Trans. Automat. Contr., vol. 37, pp. 1483-1500, 1995.

[20] _ "Smoothed perturbation analysis derivative estimation for Markov chains," Operations Res. Lett., vol. 15, pp. 241-251, 1994.

[21] A. A. Gaivoronski, L. Y. Shi, and R. S. Sreenivas, "Augmented infinitesimal perturbation analysis: An alternate explanation," Discrete Event Dynamic Syst.: Theory Appl., vol. 2, pp. 121-138, 1992.

[22] P. Glasserman, Gradient Estimation Via Perturbation Analysis. Boston: Kluwer, 1991.

[23] _ "The limiting value of derivative estimators based on perturbation analysis," Stochastic Models, vol. 6, pp. 229-257, 1990.

[24] P. W. Glynn and S. P. Meyn, "A Lyapunov bound for solutions of Poisson's equation," Ann. Probab., vol. 24, 1996.

[25] W. B. Gong and Y. C. Ho, "Smoothed perturbation analysis for discrete event dynamic systems," IEEE Trans. Automat. Contr., vol. 32, pp. 858-866, 1987.

[26] A. Haurie, P. L'Ecuyer, and C. van Delft, "Convergence of stochastic approximation coupled with perturbation analysis in a class of manufacturing flow control models," Discrete Event Dynamic Syst.: Theory Appl., vol. 4, pp. 87-111, 1994.

[27] O. Hernandez-Lerma and J. B. Lasserre, "Existence of solution to the Poisson equation in $L_{p}$ spaces," in Proc. 35rd IEEE Conf. Decision Contr., 1996.

[28] P. Heidelberger, X. R. Cao, M. Zazanis, and R. Suri, "Convergence properties of infinitesimal perturbation analysis estimates," Management Sci., vol. 34, no. 11, pp. 1281-1302, 1988.

[29] Y. C. Ho and X. R. Cao, Perturbation Analysis of Discrete-Event Dynamic Systems. Boston: Kluwer, 1991.

[30] Y. C. Ho and S. Li, "Extensions of perturbation analysis of discrete event dynamic systems," IEEE Trans. Automat. Contr., vol. 33, pp. 427-438, 1988.

[31] Y. C. Ho, X. R. Cao, and C. G. Cassandras, "Infinitesimal and finite perturbation analysis for queueing networks," Automatica, vol. 19, pp. 439-445, 1983.

[32] A. Jensen, "Markov chains as an aid in the study of Markov processes," Skandinavian Aktuartidskr, vol. 36, pp. 87-91, 1953.

[33] J. G. Kemeny and J. L. Snell, Finite Markov Chains. New York: Van Nostrand, 1960.

[34] _ , "Potentials for denumerable Markov chains," J. Math. Anal. Appl., vol. 3, pp. 196-260, 1961.

[35] A. M. Makowski and A. Shwartz, "On the Poisson equation for countable Markov chains: Existence of solutions and parameter dependence by probabilistic methods," Inst. Syst. Res., Univ. Maryland, College Park, MD, Tech. Rep. T.R. 94-87.

[36] C. D. Meyer, Jr., "The role of the group generalized inverse in the theory of finite Markov chains," SIAM Rev., vol. 17, pp. 443-464, 1975. 
[37] S. P. Meyn and R. L. Tweedie, "Generalized resolvents and Harris recurrence of Markov processes," Contemporary Math., vol. 149, pp 227-250, 1993

[38] Markov Chains and Stochastic Stability. London: SpringerVerlag, 1993

[39] _ "Stability of Markovian processes II: Continuous time processes and sampled chains," Adv. Appl. Probab., vol. 25, pp. 487-517, 1993.

[40] _ "Stability of Markovian processes III: Foster-Lyapunov criteria for continuous time processes," Adv. Appl. Probab., vol. 25, pp. 518-548, 1993.

[41] D. Revuz, Markov Chains. Amsterdam: North-Holland, 1984.

[42] D. J. S. Robison, A Course in the Theory of Groups. New York: Springer-Verlag, 1993.

[43] S. Rolewicz, Functional Analysis and Control Theory. $\quad$ D. Reidel, 1987.

[44] P. J. Schweitzer, "Perturbation theory and finite Markov chains," J. Appl. Probab., vol. 5, pp. 401-413, 1968.

[45] E. Seneta, Non-Negative Matrices and Markov Chains. New York: Springer-Verlag, 1981.

[46] ㄴ "Sensitivity analysis, ergodicity coefficients, and rank-one updates for finite Markov chains," Numerical Solution Markov Chains, W. J. Steward, Ed. New York: Marcel Dekker, 1991, pp. 121-129.

[47] R. Suri, "Perturbation analysis: The state of the art and research issues explained via the G/G/1 queue," Proc. IEEE, vol. 77, pp. 114-138, 1989.

[48] R. Suri and M. A. Zazanis, "Perturbation analysis gives strongly consistent sensitivity estimates for the M/G/1 queue," Management Sci. vol. 34, pp. 39-64, 1988.
[49] Q. Y. Tang and H. F. Chen, "Convergence of perturbation analysis based optimization algorithm with fixed number of customers period," Discrete Event Dynamic Systems: Theory Appl., vol. 4, pp. 359-375, 1994.

[50] P. Vakili, "A standard clock technique for efficient simulation," Operations Res. Lett., vol. 10, pp. 445-452, 1991.

[51] N. Xiao, F. F. Wu, and S. M. Lun, "Dynamic bandwidth allocation using infinitesimal perturbation analysis," IEEE Infocom'94, pp. 383-389.

[52] H. Yan and X. Y. Zhou, "Finding optimal number of Kanbans in a manufacturing system via perturbation analysis," Lecture Notes in Control and Information Sciences, vol. 199. New York: SpringerVerlag, 1994, pp. 572-578.

Xi-Ren Cao (S'82-M'84-SM'89-F'96), for photograph and biography, see p. 1229 of the September 1997 issue of this TRANSACTIONS.

Han-Fu Chen (SM'94-F'97), for photograph and biography, see p. 1229 of the September 1997 issue of this TransaCTIONS. 